\title{
Is elevated triglyceride high density lipoprotein cholesterol ratio a risk factor that causes acute coronary syndrome to appear earlier?
}

\author{
Dogan C, Bayram Z, Karagoz A, Bakal RB, Erdogan E, Yilmaz F, Akbal OY, Acar RD, \\ Aktemur T, Guvendi B, Karaduman A, Hakgor A, Kaymaz C, Ozdemir N
}

Kartal Kosuyolu Research and Education Hospital, Kartal, Istanbul, Turkey. draliko@yahoo.com

\begin{abstract}
BACKGROUND: The purpose of this study was to assess the relationship between the triglyceride/high density lipoprotein cholesterol ratio and the risk of acute myocardial infarction in young adults.

PATIENTS AND METHODS: A total of 621 patients, who underwent coronary angiography (CAG) due to Myocardial Infarction (MI) at our hospital were included in this study. Demographic characteristics, risk factor profile, laboratory test results, electrocardiographic and CAG findings were assessed in the selected groups.

RESULTS: Total cholesterol, triglyceride/high density lipoprotein cholesterol (Tg/HDL) ratio, Tg levels, were higher in younger patients with MI, while glucose and high-density lipoprotein levels were lower. Using propensity score matching in the matched population comparing young patients to the older ones, serum triglyceride levels [179 (145-231) vs 148 (101-197)] and triglyceride to high density lipoprotein cholesterol ratio [5.8 (4.1-9.1) vs 3.0 (1.8-4.6)] were significantly higher, whereas high density lipoprotein levels were observed dramatically lower $(32.6 \pm 8.2$ vs $41.7 \pm 8.8)$.

CONCLUSION: This study demonstrated that $\mathrm{Tg} / \mathrm{HDL}$ ratio may be an important predictor for an acute coronary syndrome in the young adult population. Tg/HDL ratio can be used to prevent MI in young adults (Tab. 3, Fig. 1, Ref. 32.) Text in PDF www.elis.sk.

KEY WORDS: acute myocardial infarction in young adults, triglyceride, HDL cholesterol.
\end{abstract}

\section{Introduction}

Coronary artery disease (CAD) is relatively rare among young individuals. Based on the age cut-off defined in studies, young patients with acute coronary syndrome account for $0.4-19 \%$ of acute coronary syndrome (ACS) cases (1-5). Despite the lower incidence in young individuals, it is important to prevent and treat $\mathrm{CAD}$ in these patients in order to achieve long life expectancy.

In patients with ACS, who had low-density lipoprotein (LDL) levels below $130 \mathrm{mg} / \mathrm{dL}$, triglycerides ( $\mathrm{Tg}$ ) were found to be higher and high-density lipoprotein (HDL) was lower compared to the control group (6). Gaziano et al showed for the first time that $\mathrm{Tg}$ / HDL ratio was a strong predictor of myocardial infarction (MI) (7). Furthermore, Tg/HDL ratio is closely associated with cardiovascular risk factors and may predict CAD development and cardiovascular mortality $(8,9)$. $\mathrm{Tg} / \mathrm{HDL}$ ratio is associated with insulin resistance and insulin resistance is known to accelerate atherogenesis. $\mathrm{Tg} / \mathrm{HDL}$ ratio also reflects the balance between atherogenic and protective lipoproteins.

Kartal Kosuyolu Research and Education Hospital, Kartal, Istanbul, Turkey Address for correspondence: A. Karagoz, MD, Kartal Kosuyolu Yuksek Ihtisas Egitim ve Arastirma hastanesi Denizer caddesi Cevizli Kavsagi No: 2, Kartal, Istanbul, Turkey .
The effect of $\mathrm{Tg} / \mathrm{HDL}$ ratio on acute myocardial infarction (AMI) occurrence in young patient groups remains currently unknown. In the present study, we investigated the role of increased $\mathrm{Tg} / \mathrm{HDL}$ on AMI occurrence in young age by comparing lipid panels of young AMI patients versus AMI patients that are older age.

\section{Patients and methods}

The patients were stratified into two groups by their age, those that were older than 50 years were put into the older patient group and those that were younger than 50 years old were put into younger patient group. The present retrospective study included 621 patients diagnosed with AMI [199 non-ST-segment elevation myocardial infarction (non-STEMI), and 422 ST-segment elevation myocardial infarction (STEMI)]. AMI cases were selected from admission to our emergency department, who underwent percutaneous coronary intervention between December 2015 and July 2016. Coronary angiographies were performed with standard Seldinger methods through femoral access. Acute myocardial infarction is defined: when there is acute myocardial injury with clinical evidence of acute myocardial ischemia and with detection of a rise and/or fall of cTn values with at least one value above the 99th percentile URL and at least one of the following: Symptoms of myocardial ischemia, New ischemic ECG changes, Develop- 
ment of pathological Q waves, Imaging evidence of new loss of viable myocardium or new regional wall motion abnormality in a pattern consistent with an ischemic etiology.

Exclusion criteria included previous CAD diagnosis, percutaneous or surgical revascularization, myocardial infarction normal coronary artery (MINOCA), previous treatment with any antihyperlipidemic agent, age $<18$ years, New York Heart Association Class III and Class IV heart failure, ongoing systemic inflammatory disease, renal or hepatic dysfunction, significant valvular disease, myocarditis, cardiomyopathies, malignancy and other pathologies that cause secondary hypertriglyceridemia.

\section{Data collection}

The clinical and demographic properties of the patients were recorded from hospital files and computer records. Baseline demographic parameters and glucose, urea, creatinine, troponin I were obtained during admission to the hospital. After an overnight fasting, blood samples for cholesterol profile were obtained within $24 \mathrm{~h}$ of admission to hospital. The blood samples for lipid profiles were analyzed using standard methods, without any delay. ECG was performed on admission and immediately after the procedure.

Baseline variables of the patients including age, gender, height, weight, and cardiovascular risk factors such as: hypertension, diabetes mellitus, dyslipidemia, current smoking, and renal failure were recorded from hospital files and computer records. Laboratory findings including lipid profile and cardiac markers, biochemistry, hemogram were recorded. The Tg/HDL-C ratio was calculated as $\mathrm{Tg}(\mathrm{mg} / \mathrm{dL})$ divided by HDL-C (mg/dL).

The patient's information was collected from hospital files and computer records including medical history, diabetes, high blood pressure, body mass index, cigarette consumption. High blood pressure was defined as being on blood pressure medication or over the $\geq 130 / 80$ by ambulatory blood pressure or over $\geq 140 / 90$ as described. Body mass index was calculated as (weight in kilograms) / (height in meters) ${ }^{2}$. Diagnosis of diabetes mellitus was defined as fasting plasma glucose $>126 \mathrm{mg} / \mathrm{dL}$; or 2-hour plasma glucose $>200 \mathrm{mg} / \mathrm{dL}$ during an oral glucose tolerance test; or in a patient with classic symptoms of hyperglycemia or hyperglycemic crisis, a random plasma glucose $>200 \mathrm{mg} / \mathrm{dL}$, a previously established diagnosis or previous treatment was also accepted. Smoking was determined as current or past history of smoking.

We performed the study according to declaration of Helsinki and this study was approved by the Local Medical Ethics Committee. Written informed consent was obtained from all the patients as needed before starting the study.

\section{Statistical analysis}

Continuous variables are expressed as the mean \pm standard deviation. Categorical variables are expressed as percentages. To compare parametric continuous variables, Student's t-test was used, and the Mann-Whitney U was used to compare nonparametric continuous variables. The chi-square test was employed to compare categorical variables. Since the study was non-randomized and was a retrospective study, we used propensity score matching method in order to minimize selection bias. In order to balance pa- tient characteristics and to generate a propensity-matched analysis for the 2 groups, a propensity score was created for the variables that are frequently used by clinicians to predict acute coronary syndromes (gender, diabetes, hypertension, smoking, MI type and family history of CAD). The propensity score was developed using SPSS, version 22.0 (IBM Corp., Armonk, NY, USA) for Windows and the R statistical package, $\mathrm{R}$ version 3.2.3 (R Foundation for Statistical Computing, Vienna, Austria). Two-tailed $\mathrm{p}<0.05$ were considered to indicate a statistical significance.

\section{Results}

The present study median Tg/HDL ratio of all patients enrolled in the study was 4.1 (2.6-5.9). Key clinical properties and laboratory results of all patients included in the study are summarized in (Tab. 1).

Baseline characteristics of younger and older MI groups are presented in Table 2. The unmatched analysis revealed that incidence of the classic risk factors, hypertension and diabetes mellitus, was lower among young patients with MI whereas smoking and family history of CAD were more frequent compared to the older patient group. Laboratory investigations indicated that total cholesterol, Tg levels, Tg/HDL ratio (Fig. 1) were higher in younger patients with MI while glucose and HDL levels were lower.

A propensity score matching was created with variables frequently used by clinicians, when predicting an acute coronary syn-

Tab. 1. Demographic properties, comorbidities and basic laboratory results of whole study's patients.

\begin{tabular}{|c|c|}
\hline \multicolumn{2}{|l|}{ Demographics } \\
\hline Age (years) & $54(44-63)$ \\
\hline Sex $($ male $)(\%)$ & 79.9 \\
\hline Body mass index & $27.3(24.5-30.1)$ \\
\hline \multicolumn{2}{|l|}{ Comorbidities } \\
\hline Hypertension (\%) & 42.7 \\
\hline Diabetes Mellitus (\%) & 26.6 \\
\hline COPD (\%) & 1.0 \\
\hline Smoking (\%) & 62.5 \\
\hline Family history of CAD (\%) & 34.0 \\
\hline \multicolumn{2}{|l|}{ Baseline laboratory results } \\
\hline Peak troponin I (ng/ml) & $8.5(1.4-35.0)$ \\
\hline Urea (mg/dl) & $35.0(28.0-41.0)$ \\
\hline Creatinine (mg/dl) & $0.8(0.7-1.0)$ \\
\hline Total cholesterol (mg/dl) & $184.0(157.0-217.0)$ \\
\hline $\operatorname{LDL}(\mathrm{mg} / \mathrm{dl})$ & $113.0(91.5-137.0)$ \\
\hline HDL (mg/dl) & $38.0(32.0-44.5)$ \\
\hline Triglyceride (mg/dl) & $153.0(109.0-197.0)$ \\
\hline Glucose (mg/dl) & $110.0(95.7-139.0)$ \\
\hline Triglyceride/HDL ratio & $4.1(2.6-5.9)$ \\
\hline $\mathrm{WBC}\left(10^{3} / \mu 1\right)$ & $10.3(8.6-13.0)$ \\
\hline Hemoglobin $(\mathrm{g} / \mathrm{dl})$ & $13.6(12.2-14.8)$ \\
\hline Platelet $\left(10^{3} / \mu 1\right)$ & $233.0(193.0-287.0)$ \\
\hline STEMI ( $\mathrm{n}$ and $\%$ ) & $422-68.0 \%$ \\
\hline NONSTEMI (n and \%) & $199-32.0 \%$ \\
\hline
\end{tabular}

CAD - coronary artery disease, COPD - chronic obstructive pulmonary disease, HDL - high density lipoprotein, NONSTEMI - non ST-segment elevation myocardial infarction, LDL - low density lipoprotein, STEMI - ST-segment elevation myocardial infarction, WBC - white blood cell. Data are expressed as the median (25th-75th percentages) and percentage (\%). 
$770-775$

Tab. 2. Basic characteristics of young and older myocardial infarction patients before propensity matching.

\begin{tabular}{|c|c|c|c|}
\hline Pre match & & & \\
\hline \multirow[b]{2}{*}{ Baseline clinical properties } & Young MI & Older MI & $\mathrm{p}$ \\
\hline & & & \\
\hline Age (years) & $42.0(38.0-46.0)$ & $61.0(55.0-68.0)$ & $<0.001$ \\
\hline Sex (male) & 85.9 & 76.2 & 0.004 \\
\hline Body mass index & $27.3(25.3-30.3)$ & $27.4(24.4-30.1)$ & 0.624 \\
\hline Hypertension (\%) & 22.6 & 54.8 & $<0.01$ \\
\hline Diabetes Mellitus (\%) & 16.2 & 32.8 & $<0.01$ \\
\hline COPD (\%) & 0.4 & 1.3 & 0.215 \\
\hline Smoking (\%) & 80.8 & 51.4 & $<0.01$ \\
\hline Family history of CAD (\%) & 47.0 & 26.1 & $<0.01$ \\
\hline \multicolumn{4}{|l|}{ Clinical presentation } \\
\hline Anterior STEMI & 57.4 & 47.0 & \\
\hline İnferior STEMI & 26.5 & 24.4 & 0.152 \\
\hline NONSTEMI & 16.1 & 18.6 & \\
\hline \multicolumn{4}{|l|}{ Baseline laboratory results } \\
\hline Peak troponin I (ng/ml) & $11.5(2.5-49.2)$ & $8.3(1.1-31.0)$ & 0.207 \\
\hline Urea (mg/dl) & $30.9(24.0-36.0)$ & $36.4(29.8-43.0)$ & $<0.001$ \\
\hline Creatinine (mg/dl) & $0.8(0.7-0.9)$ & $0.8(0.7-1.0)$ & 0.002 \\
\hline Total cholesterol (mg/dl) & $189.5(159.0-225.2)$ & $182.0(156.0-213.0)$ & 0.047 \\
\hline $\mathrm{LDL}(\mathrm{mg} / \mathrm{dl})$ & $117.0(92.0-140.0)$ & $112.0(91.0-135.0)$ & 0.175 \\
\hline HDL (mg/dl) & $32.0(26.0-38.0)$ & $40.0(35.0-47.0)$ & $<0.001$ \\
\hline Triglyceride (mg/dl) & $181.0(145.0-247.0)$ & $129.0(88.0-173.0)$ & $<0.001$ \\
\hline Glucose (mg/dl) & $104.0(92.0-128.0)$ & $114.0(100.0-151.0)$ & $<0.001$ \\
\hline Triglyceride/HDL ratio & $5.7(4.2-9.1)$ & $3.1(2.0-4.6)$ & $<0.001$ \\
\hline WBC $\left(10^{3} / \mu 1\right)$ & $11.0(9.0-13.3)$ & $10.0(8.3-12.4)$ & 0.002 \\
\hline Hemoglobin (g/dl) & $14.0(12.9-15.0)$ & $13.2(12.0-14.5)$ & $<0.001$ \\
\hline Platelet $\left(10^{3} / \mu 1\right)$ & $252(214.0-301.0)$ & $220.0(183.0-274.0)$ & $<0.001$ \\
\hline
\end{tabular}

CAD - coronary artery disease, COPD - chronic obstructive pulmonary disease, HDL - high density lipoprotein, LDL - low density lipoprotein, NONSTEMI - non STsegment elevation myocardial infarction, STEMI - ST-segment elevation myocardial infarction, WBC - white blood cell. Data are expressed as the median (25th-75th percentages) and percentage (\%). $\mathrm{p}<0.05$ was considered statistically significant.

drome risk for individual patients (gender, diabetes, hypertension, smoking, MI type and family history of CAD) in order to balance patient characteristics and to generate a propensity-matched analysis for the 2 groups (Tab. 3). In the matched population, while

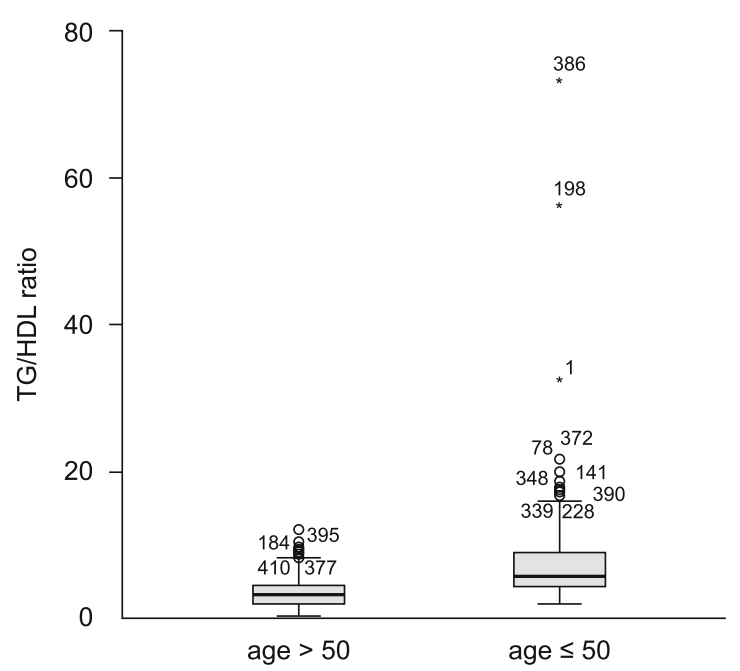

Fig. 1. Triglyceride high density lipoprotein ratio (Tg/HDL) of young and older myocardial infarction patients. The $x$-axis of this graph shows the $\mathrm{Tg} / \mathrm{HDL}$ ratios and the $\mathrm{y}$-axis shows the age groups $($ age $<$ 50 as young, age $\geq 50$ as older patients). young MI patients were compared to older MI patients, serum Tg levels [179 (145-231) vs 148 (101-197)] and Tg/HDL ratio [5.8 (4.1-9.1) vs 3.0 (1.8-4.6)] were significantly higher whereas HDL levels were observed as dramatically lower $(32.6 \pm 8.2$ vs $41.7 \pm 8.8$ ).

The analysis performed after propensity score matching revealed that there was a significant difference between young and older MI groups in terms of Tg, HDL levels and Tg/HDL ratio.

\section{Discussion}

The results of the present study indicated that increased $\mathrm{Tg} /$ HDL ratio was associated with the risk of myocardial infarction in young adults based on the propensity score analysis. The risk of MI in young individuals with high $\mathrm{Tg} / \mathrm{HDL}$ ratio was increased regardless of serum total cholesterol levels and low-density lipoprotein.

$\mathrm{Tg}$ rich lipoprotein cholesterol in post hoc analysis of TNT study showed that patients with coronary artery disease, who had higher TG rich lipoprotein levels were at a higher risk of major cardiac adverse events even if they had the same LDL levels (10 circ). Latest studies showed that in addition to lowering TG levels, trying to increase the HDL levels in treatment might not be as useful as previously thought, it might even worsen the treatment (11-13). Subtyping the HDL levels and planning treatment according to HDL subtype may prove better results in near future. (14). These results showed that TG or HDL might not be the only 
Tab. 3. Basic characteristics of young and older myocardial infarction patients after propensity matching.

\begin{tabular}{|c|c|c|c|}
\hline \multicolumn{4}{|l|}{ Post match } \\
\hline & Young MI (n:154) & Older MI (n: 154) & $\mathrm{p}$ \\
\hline \multicolumn{4}{|l|}{ Baseline clinical properties } \\
\hline Age (years) & $40.8 \pm 6.2$ & $60.4 \pm 7.4$ & $<0.001$ \\
\hline Sex (male) & 85.1 & 85.1 & 1.000 \\
\hline Body mass index & $27.4 \pm 4.6$ & $25.7 \pm 4.2$ & 0.017 \\
\hline Hypertension (\%) & 29.2 & 28.6 & 0.900 \\
\hline Diabetes Mellitus (\%) & 19.5 & 18.8 & 0.885 \\
\hline COPD (\%) & 0.8 & 1.7 & 0.511 \\
\hline Smoking (\%) & 77.9 & 77.3 & 0.891 \\
\hline Family history of CAD (\%) & 35.7 & 36.4 & 0.906 \\
\hline \multicolumn{4}{|l|}{ Clinical presentation } \\
\hline Anterior STEMI & 37 & 36.4 & 0.989 \\
\hline İnferior STEMI & 40.3 & 40.3 & \\
\hline NONSTEMI & 22.7 & 23.4 & \\
\hline \multicolumn{4}{|l|}{ Baseline laboratory results } \\
\hline Peak troponin I (ng/ml) & $6.9(1.2-18.6)$ & $14.5(1.92-50.0)$ & 0.114 \\
\hline Urea (mg/dl) & $29.3 \pm 8.1$ & $35.0 \pm 11$ & 0.002 \\
\hline Creatinine (mg/dl) & $0.76 \pm 0.15$ & $0.79 \pm 0.20$ & 0.486 \\
\hline Total cholesterol (mg/dl) & $194.0 \pm 49.0$ & $187.0 \pm 42.0$ & 0.211 \\
\hline $\mathrm{LDL}(\mathrm{mg} / \mathrm{dl})$ & $123(94-153)$ & $119(98-141)$ & 0.267 \\
\hline $\operatorname{HDL}(\mathrm{mg} / \mathrm{dl})$ & $32.6 \pm 8.2$ & $41.7 \pm 8.8$ & $<0.001$ \\
\hline Triglyceride (mg/dl) & $179(145-231)$ & $148(101-197)$ & $<0.001$ \\
\hline Glucose (mg/dl) & $122.0 \pm 58$ & $137.0 \pm 69$ & 0.001 \\
\hline Triglyceride/HDL ratio & $5.8(4.1-9.1)$ & $3.0(1.8-4.6)$ & $<0.001$ \\
\hline WBC $\left(10^{3} / \mu 1\right)$ & $10.9 \pm 3.1$ & $10.9 \pm 3.7$ & 0.852 \\
\hline Hemoglobin (g/dl) & $13.8 \pm 1.7$ & $13.4 \pm 1.7$ & 0.023 \\
\hline Platelet $\left(10^{3} / \mu 1\right)$ & $262.0 \pm 88.0$ & $235.0 \pm 78$ & 0.003 \\
\hline
\end{tabular}

CAD - coronary artery disease, COPD - chronic obstructive pulmonary disease, HDL - high density lipoprotein, LDL - low density lipoprotein, NONSTEMI - non STsegment elevation myocardial infarction, STEMI - ST-segment elevation myocardial infarction, WBC - white blood cell. Data are expressed as the median (25th-75th percentages), the mean \pm standard deviation and percentage $(\%)$. $\mathrm{p}<0.05$ was considered statistically significant.

treatment parameters and maybe statins combined with pcsk9 inhibitor or ezetimib will allow us to reach the LDL goal or reduce the relative risk especially in young adults.

With such an active role in atherogenesis, the Tg/HDL ratio is known to predict cardiovascular events both in healthy individuals and patients with CAD. In healthy individuals, the relationship between this ratio and insulin resistance, cardiometabolic risk and cardiovascular disease was shown in the previous studies $(15,16-19)$.

When Tg and HDL are considered separately, increased Tg and decreased HDL was shown to predict cardiovascular disease, independent from LDL levels (20).

Determining the risk factors and adequate management is one of the corner stones of preventing coronary artery disease (CAD). Age, smoking, hypertension, diabetes mellitus, hypercholesterolemia are the most acclaimed risk factors; however, they may fall short when determining the definite cardiovascular risk in young adults. Atherogenesis, which is the most common cause of CAD pathogenesis, has an established association with dyslipidemia. Management of dyslipidemia targets the apo-B containing LDL (21). In patients with high risk, a residual cardiovascular risk persists despite a successful LDL lowering with statins $(22,23) . \mathrm{Tg} /$ HDL ratio appears as a valuable parameter for identifying this residual risk. $\mathrm{Tg} / \mathrm{HDL}$ ratio has been shown to be a strong predictor of cardiovascular disease, independent from age, smoking, hypertension, diabetes mellitus and cholesterol (15).
Typical plaque rupture is rare in young patients with ACS (24, 25); therefore, other risk factors apart from the conventional risk factors should be taken into account in these patients. Smoking, family history, obesity and hypercholesterolemia were more common in our patient group of young MI cases. In the lipid analysis, particularly $\mathrm{Tg}$ and total cholesterol levels were distinctly higher, while HDL levels were significantly lower, and no difference was observed in terms of LDL levels. These findings are consistent with the studies reporting that insulin and hypertriglyceridemia are also important risk factors in patients $<50$ years of age while smoking, obesity, hypercholesterolemia and male gender tend to be more common (26). In young patients with ACS, hypertriglyceridemia has been associated with premature CAD (27-29) and $\mathrm{Tg} / \mathrm{HDL}$ ratio was also held responsible for increased arterial stiffness (30). Although the mechanisms leading to the effect on the pathogenesis of atherosclerosis are currently unclear, some relevant mechanisms of Tg/HDL ratio are; Tg/HDL ratio is associated with insulin resistance, reflects the balance between atherogenic and protective lipoproteins $(31,32)$ and also $\mathrm{Tg} / \mathrm{HDL}$ ratio triggers inflammatory mechanisms.

In our study, despite the fact that LDL was not different in the young MI group, the higher Tg/HDL ratio and lower HDL levels were critical and essential findings. Our findings are supported by studies, which demonstrated that higher triglyceride levels and lower HDL levels are common in patients with ACS. However, previous studies demonstrated that patients with lower HDL and 


\section{$770-775$}

higher TG levels are still under higher ACS risk even though they receive a lipid-lowering treatment, when compared to the control group. Tg or HDL alone are significant predictors of ACS in patients with cardiovascular disease (20), the combination of these parameters as $\mathrm{Tg} / \mathrm{HDL}$ may be more valuable than utilizing these two separately in order to determine the cardiovascular risk in these patients. Taken together, the present study results suggest that in young individuals $\mathrm{Tg} / \mathrm{HDL}$ are not only a cardiovascular risk marker, but also a potential target for therapeutic intervention.

\section{Conclusion}

In our study, our findings have given us the idea that the $\mathrm{Tg} /$ HDL ratio can be used to predict the occurrence of acute coronary syndrome in young adults. Especially in adults under the age of $50, \mathrm{Tg} / \mathrm{HDL}$ ratio may be determined and necessary precautions might be taken to prevent ACS.

\section{Limitations}

The number of patients in our single-centered study is relatively low. Since it was not a prospective study, no patients were followed up and healthy people were not taken as a control group. Increased thrombogenicity associated with an increased susceptibility to acute coronary syndrome in young people has not been examined for genetic and laboratory tests. During first week of acute myocardial infarction, lipid levels may be measured differently, however, since both of the groups' lipid levels were obtained at the same time frame after the infarction, the difference effects both groups.

\section{References}

1. Wolfe MW, Vacek JL. Myocardial infarction in the young. Angiographic features and risk factor analysis of patients with myocardial infarction at or before the age of 35 years. Chest 1988; 94: 926-930.

2. Gotsman I, Lotan C, Mosseri M. Clinical manifestations and outcome of acute myocardial infarction in very young patients. Isr Med Assoc J 2003; 5: 633-636.

3. Morillas P, Bertomeu V, Pabon $\mathbf{P}$ et al. Characteristics and outcome of acute myocardial infarction in young patients. The PRIAMHO II study. Cardiology 2007; 107: 217-225.

4. Hoit BD, Gilpin EA, Henning $\mathbf{H}$ et al. Myocardial infarction in young patients: an analysis by age subsets. Circulation 1986; 74: 712-721.

5. Doughipertansiony M, Mehipertansiona R, Bruckman D et al. Acute myocardial infarction in the young-the University of Michigan experience. Am Heart J 2002; 143: 56-62.

6. Carey VJ, Bishop L, Laranjo $\mathbf{N}$ et al. Contribution of high plasma triglycerides and low high density lipoprotein cholesterol to residual risk of coronary heart disease after establishment of low-density lipoprotein cholestero lcontrol. Am J Cardiol. 2010; 106: 757-763.

7. Gaziano JM, Hennekens CH, O'Donnell CJ et al. Fasting triglycerides, high-density lipoprotein, and risk of myocardial infarction. Circulation. 1997; 96: 2520-2525.
8. De Leon AC, Coello SD, Gonzalez DA et al. Impaired fasting glucose, ancestry and waist-to-heighipertansion ratio: main predictors of incident diagnosed diabetes in the Canary Islands. Diabet Med 2012; 29: 399-403.

9. Hadaegh F, Khalili D, Ghasemi A et al. Triglyceride/HDL-cholesterol ratio is an independent predictor for coronary heart disease in a population of Iranian men. Nutr Metab Cardiovasc Dis 2009; 19: 401-408.

10. Vallejo-Vaz AJ, Fayyad R et al. Triglyceride-Rich Lipoprotein Cholesterol and Risk of Cardiovascular Events Among Patients Receiving Statin Therapy in the Treating to New Targets (TNT) Trial. Circulation. 2018 Apr 4. pii: CIRCULATIONAHA.117.032318.

11. Barter PJ, Caulfield M, Eriksson $M$ et al; ILLUMINATE Investigators. Effects of torcetrapib in patients at high risk for coronary events. N Engl J Med 2007; 357 (21): 2109-2122.

12. Lincoff AM, Nicholls SJ, Riesmeyer JS et al; ACCELERATE Investigators. Evacetrapib and Cardiovascular Outcomes in High-Risk Vascular Disease. N Engl J Med 2017; 376 (20): 1933-1942.

13. HPS3/TIMI55-REVEAL Collaborative Group, Bowman L, Hopewell JC, Chen F, Wallendszus K, Stevens W, Collins R, Wiviott SD, Cannon CP, Braunwald E, Sammons E, Landray MJ. Effects of Anacetrapib in Patients with Atherosclerotic Vascular Disease. N Engl J Med 2017; 377 (13): 1217-1227.

14. Kasko M, Kasko V, Oravec S. Would Janus' view on HDL be useful? Bratisl Lek Listy 2018; 119 (4): 245-248. doi: 10.4149/BLL_2018_046.

15. Salazar MR, Carbajal HA, Espeche WG, Leiva Sisnieguez CE, March CE, Balbin E et al. Comparison of the abilities of the plasma triglyceride/high-density lipoprotein cholesterol ratio and the metabolic syndrome to identify insulin resistance. Diab Vasc Dis Res 2013; 10 (4): $346-352$.

16. Salazar MR, Carbajal HA, Espeche WG et al. Relationships among insulin resistance, obesity, diagnosis of the metabolic syndrome and cardiometabolic risk. Diab Vasc Dis Res 2011; 8 (2): 109-116.

17. Salazar MR, Carbajal HA, Espeche WG et al. Relation among the plasma triglyceride/high-density lipoprotein cholesterol concentration ratio, insulin resistance and associated cardio-metabolic risk factors in men and women. Am J Cardiol 2012; 109: 1749-1753.

18. Salazar MR, Carbajal HA, Espeche WG et al. Cardiovascular disease risk and outcome: use of the plasma triglyceride/high-density lipoprotein cholesterol concentration ratio versus metabolic syndrome criteria. J Intern Med 2013; 273 (6): 595-601.

19. Tae Ik Chang, Elani Streja, Melissa Soohoo et al. Association of Serum Triglyceride to HDL Cholesterol Ratio with All-Cause and Cardiovascular Mortality in Incident Hemodialysis Patients. Clin J Am Soc Nephrol 2017; 12 (4): 591-602. doi: 10.2215/CJN.08730816.

20. Péter Koncsos, Péter Fülöp, Imre Juhász et al. Changes in triglyceride, HDL-C, and non-HDL-C levels in patients with acute coronary syndrome. Wien Klin Wschr 2016; 128 (23-24): 858-863.

21. Reiner Z, Catapano AL, De Backer G. European Association for Cardiovascular Prevention \& Rehabilitation et al. ESC/EAS Guidelines for the management of dyslipidaemias: the Task Force for the management of dyslipidaemiasof the European Society of Cardiology (ESC) and the European Atherosclerosis Society (EAS). EurHeart J 2011; 32: 1769-1818.

22. Baigent C, Keech A, Kearney PM. Cholesterol Treatment Trialists' (CTT) Collaborators et al. Efficacy and safety of cholesterol-loweringtreatment: prospectivemeta-analysis of data from 90,056 participants in 14 randomised trials of statins. Lancet 2005 ; 366: 1267-1278. 
23. Kearney PM, Blackwell L, Collins R. Cholesterol Treatment Trialists' (CTT) Collaborators et al. Efficacy of cholesterol lowering therapy in 18,686 people with diabetes in 14 randomised trials of statins: a metaanalysis. Lancet 2008; 371: 117-125.

24. Yasunori U, Katsuki O, Nobuyuki O et al. Acute Myocardial Infarction Without Disrupted Yellow Plaque in Young Patients Below 50 Years Old. J Interv Cardiol 2007; 20 (3): 177-181.

25. Puricel $\mathbf{S}$, Lehner $\mathbf{C}$, Oberhänsli $M$ et al. Acute coronary syndrome in patients younger than 30 years. Aetiologies, baseline characteristics and long term clinical outcome. Swiss Med Wkly 2013; 143: w13816. doi: 10.4414/smw.2013.13816.

26. Tsai WC, Wu Y, Lin GM et al. Clinical characteristics of patients less than forty years old with coronary artery disease in Taiwan: A crosssectional study. Acta Cardiol Sin 2017; 33 (3): 233-240.

27. Jinnouchi H, Sakakura K, Wada $\mathbf{H}$ et al. Clinical features of myocardial infarction in young Japanese patients. Int Heart J 2013; 54: $123-128$.
28. Chen L, Chester M, Kaski JC. Clinical factors and angiographic features associated with premature coronary artery disease. Chest 1995; 108: 364-369.

29. Bhardwaj R, Kandoria A, Sharma R. Myocardial infarction in young adults-risk factors and pattern of coronary aretery involvement. Niger Med J 2014; 55: 44-47.

30. Urbina EM, Khoury PR, McCoy CE et al. Triglyceride to HDL-C ratio and increased arterial stiffness in children, adolescents, and young adults. Pediatrics 2013; 131 (4): e1082Ye1089.

31. Dobiasova M, Frohlich J. The plasma parameter log (TG/HDL-C) as an atherogenic index: correlation with lipoprotein particle size and esterification rate in apoB-lipoprotein-depleted plasma (FER(HDL)). Clin Biochem 2001; 34: 583-588.

32. Guerin M, Le Goff W, Lassel TS et al. Atherogenic role of elevated CE transfer from HDL to very low density lipopotein (1) and dense LDL in type 2 diabetes: impact of the degree of triglyceridemia. Arterioscler Thromb Vasc Biol 2001; 21: 282-288. 